\title{
Symmetrized models of last passage percolation and non-intersecting lattice paths
}

\author{
Peter J. Forrester and Eric M. Rains \\ *Department of Mathematics and Statistics, University of Melbourne, \\ Victoria 3010, Australia ; \\ † Department of Mathematics, University of California, Davis, CA 95616, USA \\ It has been shown that the last passage time in certain symmetrized models of directed per- \\ colation can be written in terms of averages over random matrices from the classical groups \\ $U(l), S p(2 l)$ and $O(l)$. We present a theory of such results based on non-intersecting lattice \\ paths, and integration techniques familiar from the theory of random matrices. Detailed \\ derivations of probabilities relating to two further symmetrizations are also given.
}

\section{Introduction}

There are a number of striking results linking models of stochastic processes to to random matrix theory (for a recent work of this type see [4]; for reviews see [7, 21]). As an easy to explain example, consider the following last passage percolation problem due to Hammersley. In the unit square mark in points uniformly at random according to a Poisson rate with intensity $\lambda$ (thus the probability the square contains $N$ points is equal to $\frac{\lambda^{N}}{N !} e^{-\lambda}$ ). Join points by straight line segments with the requirement that the segments have positive slope and form a continuous path, and extend this path to begin at $(0,0)$ and finish at $(1,1)$. Define the length of the extended path as the number of points it contains, and denote by $l^{\square}=l^{\square}(\lambda)$ the stochastic variable specifying the maximum of the lengths of all possible extended paths (see Figure 1). Then it is known from the work of Gessel [11] and Rains [19] (see also [5]) that

$$
\operatorname{Pr}\left(l^{\square} \leq l\right)=\left\langle\prod_{j=1}^{l} e^{\sqrt{\lambda} \cos \theta_{j}}\right\rangle_{U(l)}
$$

where the average is with respect to the eigenvalue probability density function (p.d.f.) of random matrices chosen uniformly at random from the group $U(l)$. The latter has the explicit form

$$
\frac{1}{(2 \pi)^{l} l !} \prod_{1 \leq j<k \leq l}\left|e^{i \theta_{k}}-e^{i \theta_{j}}\right|^{2}
$$

The formula (1.1) is central to the proof by Baik, Deift and Johansson [1] giving the limiting scaled distribution of the longest increasing subsequence of a random permutation.

In a substantial work Baik and Rains [2] have considered symmetrized generalizations of the Hammersley process, and have shown that the cumulative probability for the analogue of the stochastic variable $l^{\square}$ can be written as an average over the classical groups $S p(2 l), O(l)$ (for two particular symmetries), or $U(2 l), U(l) \oplus U(l)$ (for two other symmetries). Moreover, random matrix formulas were also given [3] for lattice generalizations of these processes (in the case of the original Hammersley process the lattice generalization is referred to as the Johansson model [13]). The proofs of 2 make sophisticated use of symmetric function theory, and have independent interest in that discipline. On the other hand it is reasonable to suggest that many researchers interested in directed percolation and growth processes will lack the necessary background in symmetric function theory to fully appreciate these proofs. This has motivated us to seek alternative derivations which to a large extent avoid the heavy use of symmetric 


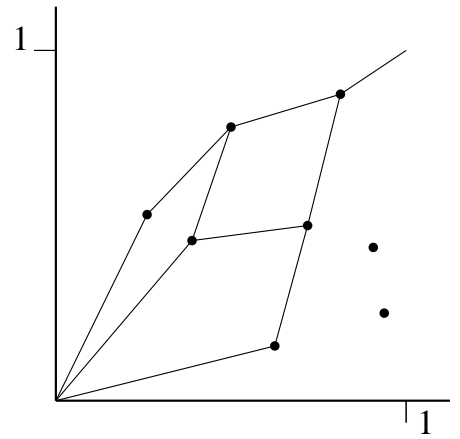

Figure 1: Eight points in the unit square, and the extended directed paths of maximum length. Since the number of segments in these paths equals four, here $l_{n}^{\square}=3$.

function theory called upon in 2. Instead our proofs make use of a non-intersecting path picture of the Johansson growth model [14, 9], an extension of this picture to a last passage percolation model with Bernoulli random variables, together with techniques familiar from the theory of random matrices (the applicability of such techniques have been foreshadowed in Section 6 of [2]). Another essential ingredient from [2] is the use of bijections from the theory of Young tableaux (see e.g. [10]). Here our presentation differs from that of 2 only in that we give more detail.

Our task then is to derive, from a non-intersecting paths picture, formulas known from [2] giving cumulative probabilities of a suitable last passage percolation variable for generalizations of the Hammersely process in terms of random matrix averages. As already mentioned, one such generalization is the Johansson model. In Section 2 its definition is recalled, as is its formulation in the non-intersecting path picture. Formulas from the theory of non-intersecting paths are then used to derive the analogue of (1.1). An analogous discussion of a variant of this model, involving Bernoulli rather than geometric random variables, is given in Section 3. The four symmetrized versions of the Johansson model are then treated separately in each of the subsequent four sections.

\section{Johansson model and polynuclear growth}

We begin by defining the last passage percolation model introduced by Johansson [13. Consider the upper right quadrant square lattice $\left\{(i, j): i, j \in \mathbb{Z}^{+}\right\}$. Associate with each lattice site $(i, j)$ a random non-negative integer variable $x_{i, j}$ chosen from the geometric distribution with parameter $a_{i} b_{j}$ so that

$$
\operatorname{Pr}\left(x_{i, j}=k\right)=\left(1-a_{i} b_{j}\right)\left(a_{i} b_{j}\right)^{k} .
$$

Denote by $(1,1) \mathrm{u} / \mathrm{rh}(n, n)$ a sequence of lattice paths starting at $(1,1)$ and finishing at $(n, n)$ with each lattice point successively connected by edges which are either directed upwards or horizontally to the right. One defines the last passage time $L_{n}^{\square}$ say as the maximum of the sum of the integer variables associated with these lattice points. Thus

$$
L_{n}^{\square}:=\max \sum_{(1,1) \mathrm{u} / \mathrm{rh}(n, n)} x_{i, j} .
$$



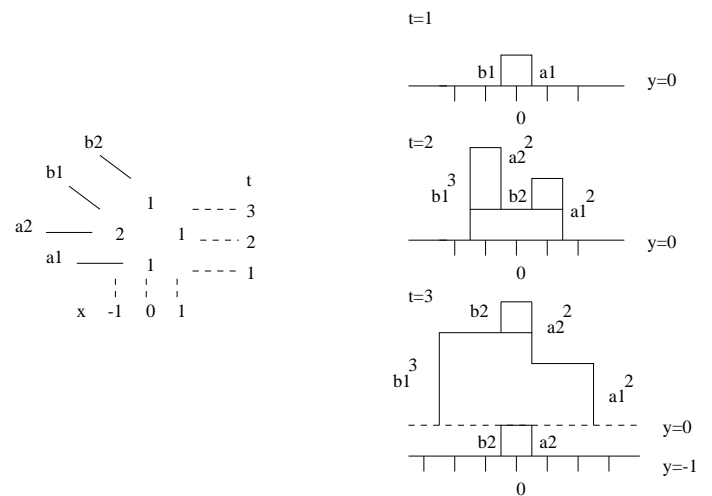

Figure 2: RSK correspondence in the polynuclear growth model picture between a weighted non-negative integer matrix and a pair of weighted non-intersecting lattice paths.

According to 2], for given parameters $\left\{a_{i}\right\},\left\{b_{j}\right\}$ the cumulative distribution can be written as a random matrix average according to

$$
\operatorname{Pr}\left(L_{n}^{\square} \leq l\right)=\prod_{i, j=1}^{n}\left(1-a_{i} b_{j}\right)\left\langle\prod_{j=1}^{n} \prod_{k=1}^{l}\left(1+a_{j} e^{-i \theta_{k}}\right)\left(1+b_{j} e^{i \theta_{k}}\right)\right\rangle_{U(l)}
$$

where the average over $U(l)$ refers to the probability density function (1.2). We seek a derivation of (2.3), and analogous formulas from [2] for symmetrized versions of the Johansson model, within a nonintersecting paths representation of the model.

Let us first revise how non-intersecting paths relate to the Johansson model [14, 9]. This is done via a geometrical construction, equivalent to the Robinson-Schensted-Knuth (RSK) correspondence from the theory of Young tableaux [10], which gives a bijection between non-negative integer matrices and pairs of non-intersecting lattice paths. Furthermore, the maximum displacement of the top-most of these paths is equal to $L_{n}^{\square}$, with the profile of this path also specifying the height profile in a statistical mechanical model referred to as the polynuclear growth model. With regard to the latter, the entries $x_{i, j}$ of an $n \times n$ non-negative integer matrix $X=\left[x_{i, j}\right]_{i, j=1, \ldots, n}$ (for convenience rows are labelled from the bottom) now represent the heights of columns of unit width centred about $x=j-i$ which occur at time $t=i+j-1$ (in labelling the matrix in terms of $x$ and $t$ it is convenient to first rotate it $45^{\circ}$ anti-clockwise). The columns are to be placed on top of the profile formed by earlier nucleation events and their growth. The right boundary of the column corresponding to $x_{i, j}$ is to be weighted $a_{i}^{x_{i, j}}$ while the left boundary is to be weighted $b_{j}^{x_{i, j}}$, and these weights are to be multiplied together with any existing weights along the same vertical segment of the profile. During each time interval the existing profile or profiles are required to grow one unit to the left and one unit to the right, with any resulting overlap, together with the corresponding portion of the weights, recorded on a profile with base one unit below. In this way a bijection between $n \times n$ integer matrices with each entry $x_{i, j}$ weighted $a_{i} b_{j}$, and a pair of weighted non-intersecting paths is obtained. A particular example is given in Figure 2

The bijection generates at most $n$ non-intersecting paths. These paths start one unit apart in the $y$-direction (at $y=0, \ldots,-(n-1))$. In the $x$-direction one member of the pair of paths starts at $x=-(2 n-3 / 2)$ and may go up in arbitrary integer amounts of a unit step at $x=-(2 n+1 / 2-2 j)$ for $j=1, \ldots, n$ with each step weighted by $b_{j}$, or to the right in steps of two units (these steps are unweighted). The other member starts at $x=(2 n-3 / 2)$ and may go up in arbitrary integer amounts of a unit step at $x=(2 n+1 / 2-2 i)$ for $i=1, \ldots, n$ with each step weighted $a_{i}$, or to the left in steps of 
two units, the latter being unweighted. Furthermore the second member is constrained so that it joins with the first member at $x=0$. Both members are equivalent to what will be termed $\mathrm{u} / \mathrm{rh}$ (up/ right horizontal) non-intersecting lattice paths. By definition such paths are defined on the square lattice and start at $x=0$, one unit apart in the $y$-direction (at $y=0, \ldots,-(n-1)$ ), and finish at $x=n-1$, with $y$-coordinates $\mu_{l}-(l-1)(l=1, \ldots, n)$ where $\mu_{1} \geq \mu_{2} \geq \cdots \geq \mu_{N} \geq 0$. The path starting at $y=-(l-1)$ is referred to as the level- $l$ path. Each path may move either up or to the right along the edges of the lattice, with each up step at $x=j-1$ weighted $q_{j}$. Define the weight of a configuration of $\mathrm{u} / \mathrm{rh}$ lattice paths as the product of all the step weights. Then it is well known (see e.g. 20]) that with $\mu:=\left(\mu_{1}, \mu_{2}, \ldots, \mu_{N}\right)$ (because of the orderings of the $\mu_{i}, \mu$ forms a partition)

$$
\left.\sum_{\substack{\text { u/rh paths } \\ \text { displacements } \mu}} \text { (weight of the paths }\right)=s_{\mu}\left(q_{1}, \ldots, q_{n}\right)
$$

where $s_{\mu}$ is the Schur polynomial.

We remark that $\mathrm{u} / \mathrm{rh}$ non-intersecting lattice paths are equivalent to semi-standard tableaux (numbered diagram of a partition $\lambda$ such that the numbers weakly increase along rows and strictly decrease down columns). Thus with $\tilde{\lambda}_{l}(j)$ denoting the number of vertical steps in the level- $l$ path at $x=j-1$, the $l$ th row of the tableaux is of length $\sum_{j=1}^{n} \tilde{\lambda}_{l}(j)=: \lambda_{l}$ and is numbered by $\tilde{\lambda}_{l}(j)$ lots of $j$ 's $(j=1, \ldots, n$ in order). An explicit example is given in Figure 3. Consequently if there are $n$ lattice paths the numbering is from the set $\{1, \ldots, n\}$ which is referred to as the content of the tableau. With $\lambda=\left(\lambda_{1}, \ldots, \lambda_{n}\right)$ denoting the partition formed from the length of the rows, the tableaux is said to have shape $\lambda$.

For future reference we note that with $\mu_{l}(n, j)$ denoting the displacement of the level- $l$ path at $x=-(2 n+1 / 2-2 j)$ as resulting from the RSK correspondence and $\mu_{l}(i, n)$ equal to the displacement of the level- $l$ path at $x=2 n+1 / 2-2 i$, we have

$$
\begin{aligned}
\sum_{l=1}^{n}\left(\mu_{l}(n, j)-\mu_{l}(n, j-1)\right) & =\sum_{i=1}^{n} x_{i, j} \\
\sum_{l=1}^{n}\left(\mu_{l}(i, n)-\mu_{l}(i-1, n)\right) & =\sum_{j=1}^{n} x_{i, j} .
\end{aligned}
$$

Also for future reference we make note of quantities generalizing $L_{n}^{\square}$ which are related to the maximum displacements $\mu_{l}$ of the level- $l$ paths for each $l=1,2, \ldots$ For this let $\left(\mathrm{rd}^{*}\right)^{l}$ denote the set of $l$ disjoint (here meaning connecting no common lattice sites) $\mathrm{rd}^{*}$ lattice paths, the latter defined as either a single point, or points connected by segments formed out of arbitrary positive integer multiples of steps to the right and steps up in the square lattice $1 \leq i, j \leq n$. Generalizing the definition (2.2) by defining

$$
L_{n}^{\square(l)}=\max \sum_{\left(\mathrm{rd}^{*}\right)^{l}} x_{i, j},
$$

a theorem of Greene [12] gives

$$
L_{n}^{\square(l)}=\sum_{m=1}^{l} \mu_{m},
$$

and thus in particular 15

$$
\mu_{1}=L_{n}^{\square(1)}:=L_{n}^{\square} .
$$

It follows from the above discussion that with the entries of the $n \times n$ matrix $X$ weighted according to (2.1), the probability that $X$ maps under the RSK correspondence to a pair of non-intersecting paths 


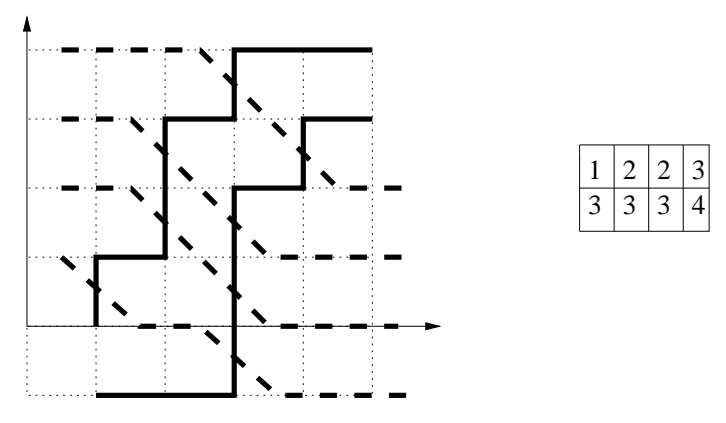

Figure 3: Drawn in heavy lines on the square lattice is a family of two $\mathrm{u} / \mathrm{rh}$ lattice paths with up segments allowed at $x=1,2,3,4$, while drawn in heavy dashed lines are the corresponding four dual lattice paths. Also given is the semi-standard tableau encoding the two $\mathrm{u} / \mathrm{rh}$ lattice paths.

with maximum displacements $\mu$ is given by 14

$$
\prod_{i, j=1}^{n}\left(1-a_{i} b_{j}\right) s_{\mu}\left(a_{1}, \ldots, a_{n}\right) s_{\mu}\left(b_{1}, \ldots, b_{n}\right)
$$

The equality (2.9) between $\mu_{1}$ and $L_{n}^{\square}$ then implies the formula [2, 14]

$$
\operatorname{Pr}\left(L_{n}^{\square} \leq l\right)=\prod_{i, j=1}^{n}\left(1-a_{i} b_{j}\right) \sum_{\mu: \mu_{1} \leq l} s_{\mu}\left(a_{1}, \ldots, a_{n}\right) s_{\mu}\left(b_{1}, \ldots, b_{n}\right) .
$$

Thus we must now show that the sum over Schur functions in (2.11) can be written as the average over $U(l)$ in (2.3). Moreover we want to achieve this task within the framework of non-intersecting paths.

An important notion for this purpose is that of the dual non-intersecting lattice paths associated with a set of $\mathrm{u} / \mathrm{rh}$ paths. The dual lattice paths connect points on the lattice $\left\{(n-1 / 2, m), n \in \mathbb{Z}_{\geq 0}, m \in \mathbb{Z}\right\}$. Points are connected by segments which are directed either right horizontal (rh) or diagonally down (dd), with a dd segment bisecting every $\mathrm{u}$ segment of the $\mathrm{u} / \mathrm{rh}$ lattice path. The dd segments are connected by rh segments to form dual lattice paths starting at $x=-1 / 2$ and finishing at $x=n-1 / 2$ in the $x$ direction, while in the $y$-direction these paths start at $y=k$ for $k=1,2, \ldots, \mu_{1}$ where $\mu_{1}$ is the maximum displacement of the level-1 path (see Figure 3). In terms of tableaux, the dual lattice paths correspond to reading down columns instead of across rows. The mapping carries over to weighted paths by simply weighting the dd segment in the dual path by the value of the $\mathrm{u}$ segment it bisects in the original $\mathrm{u} / \mathrm{rh}$ path.

The key feature for our purpose is that the constraint on the $\mathrm{u} / \mathrm{rh}$ lattice paths having maximum displacement less than or equal to $l$ translates in the dual path picture as constraining the number of paths to be less than or equal to $l$. As only dd segments are weighted we can take the number of paths as being exactly equal to $l$. Furthermore we can regard a pair of rh/dd lattice paths, each with the same end points and each containing $l$ paths but weighted from $\left\{b_{j}\right\}$ and $\left\{a_{i}\right\}$ respectively, as a single set of non-intersecting paths. In the latter the second member of the pair is reflected about $x=n-1 / 2$ so that its final positions are at $(2 n-1 / 2, k)$ for $k=1,2, \ldots, l$ at it consists of right horizontal and up diagonal segments, the latter being weighted by $a_{j}$ according to them passing through $x=2 n+1 / 2-j$. With the initial and final $y$ coordinates generalized to $y_{1}^{(0)}, \ldots, y_{l}^{(0)}$ and $y_{1}, \ldots, y_{l}$ respectively, let $G_{2 n}\left(y_{1}^{(0)}, \ldots, y_{l}^{(0)} ; y_{1}, \ldots, y_{l}\right)$ denote the total weight of all such paths. It then follows 
from the dual lattice paths interpretation of the sum in (2.11) that

$$
\operatorname{Pr}\left(L^{\square} \leq l\right)=\prod_{i, j=1}^{n}\left(1-a_{i} b_{j}\right) G_{2 n}\left(\left\{y_{j}^{(0)}=-(j-1)\right\}_{j=1, \ldots, l} ;\left\{y_{j}=-(j-1)\right\}_{j=1, \ldots, l}\right) .
$$

Because each path in the family is directed, the weight $G_{2 n}$ of all paths in the family is given in terms of the weight of a single path in the family, $g_{2 n}\left(y^{(0)} ; y\right)$ say. Thus the well known Linström-Gessel-Viennot theorem (see e.g. [16]) gives

$$
G_{2 n}\left(y_{1}^{(0)}, \ldots, y_{l}^{(0)} ; y_{1}, \ldots, y_{l}\right)=\operatorname{det}\left[g_{2 n}\left(y_{j}^{(0)} ; y_{k}\right)\right]_{j, k=1, \ldots, l} .
$$

Furthermore it is easy to see that

$$
g_{2 n}\left(y^{(0)} ; y\right)=\frac{1}{2 \pi} \int_{0}^{2 \pi} \prod_{j=1}^{N}\left(1+a_{j} e^{-i \theta}\right)\left(1+b_{j} e^{i \theta}\right) e^{-i\left(y-y^{(0)}\right) \theta} d \theta .
$$

Substituting (2.14) in (2.13), then substituting the result in (2.12) and recalling the general formula 23 ,

$$
\operatorname{det}\left[\frac{1}{2 \pi} \int_{0}^{2 \pi} a(\theta) e^{i(j-k) \theta} d \theta\right]_{j, k=1, \ldots, n}=\left\langle\prod_{l=1}^{n} a\left(\theta_{l}\right)\right\rangle_{U(n)}
$$

we see that (2.3) is reclaimed.

\section{Polynuclear growth with Bernoulli random variables}

A variant of the last passage percolation model revised in the previous section is to replace (2.1) by the Bernoulli distribution

$$
\operatorname{Pr}\left(x_{i j}=k\right)=\frac{\left(a_{i} b_{j}\right)^{k}}{1+a_{i} b_{j}}, \quad k=0,1 .
$$

Let $X=\left[x_{i, j}\right]_{\substack{i=1, \ldots, m \\ j=1, \ldots, n}}$ be an array of such variables. One specifies the corresponding last passage time by

$$
L_{m, n}^{01}:=\max \sum_{\substack{\left(i^{\prime}, j^{\prime}\right) \in \text { bottom to top } \\ \text { u/rd paths }}} x_{i^{\prime}, j^{\prime}}
$$

where the sum is over all $\mathrm{u} / \mathrm{rd}$ paths in the rectangle $1 \leq i^{\prime} \leq m, 1 \leq j^{\prime} \leq n$ from the bottom row (row 1) to the top row (row $m$ ). The segments of the path join entries successively to the north or north-east in the array.

Underlying this model is the dual RSK correspondence [10]. To our knowledge this has not previously been related to a layered growth model formed out of non-intersecting paths. Here such a relationship will be presented.

Again, the entries $x_{i j}$ of the array $X$ are regarded as recording nucleation events. However, unlike the situation with the RSK correspondence itself, the entries of $X$ are not first rotated $45^{\circ}$ before being associated with positions and times. Rather $x_{i, j}=1$ denotes a nucleation event (a unit square) which is positioned above the segment $x=j-1$ to $x=j$, and on top of earlier nucleation events and their growth (this is in common with the polynuclear growth model of the previous section). These nucleation events occur at successive times $t=1,2, \ldots, n$, with the positions recorded by 1 's in the corresponding rows of $X$. Thus to begin, at $t=1$ the nucleation events are read off from the first row of $X$ and marked on the line $y=0$. As $t \mapsto t+1$, the existing profile(s) is to grow one unit to the right (but not the left) until it joins up with the neighbouring nucleation event on the right. If there is no such right neighbour, and 

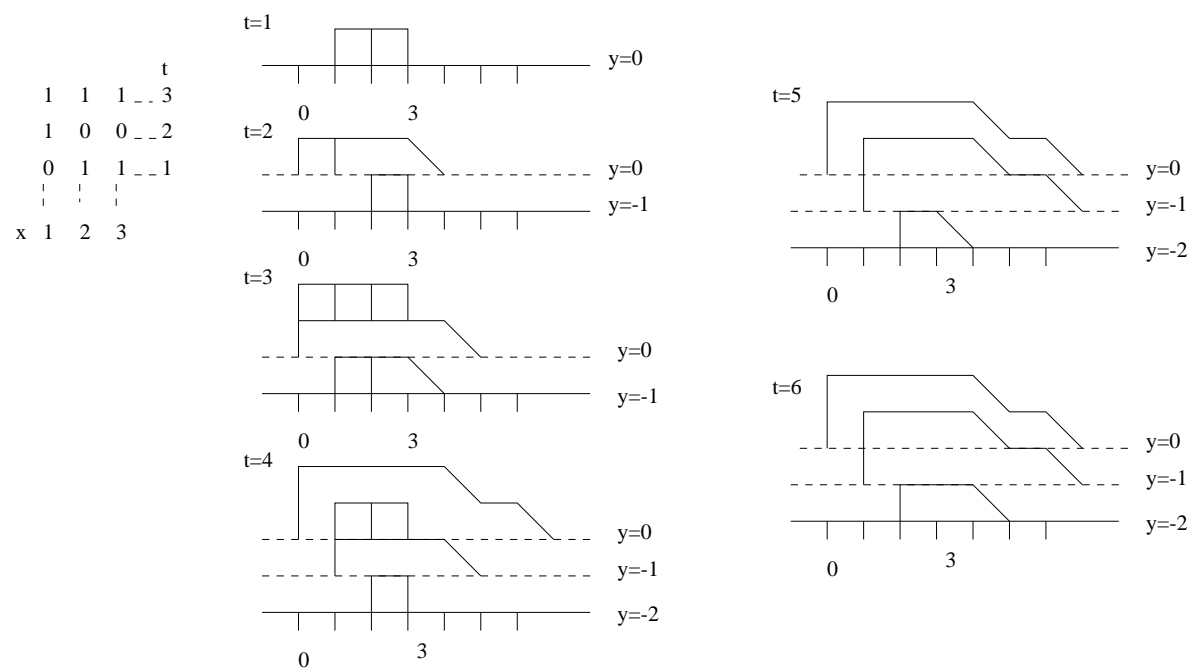

Figure 4: Mapping from a 0,1 matrix to a pair of non-intersecting lattice paths.

this nucleation event is yet to grow (i.e. recorded in the previous time step), it is to grow to $x=n+1$ and have its shape modified by removing the upper triangular half of its final square. If it has right edge at $x \geq n+1$, it is to grow one unit to the right. Also, the meeting of all nucleation events in going from $t \mapsto t+1$ are to be recorded on the line $y=-t$ as new nucleation events with left edge at the positions of the meetings.

This procedure is to stop after time $m+l$ along $y=-(l-1)(l=1, \ldots, m+1)$, this being the maximum time for which new nucleation events can be created and then grow once. The layers of profiles which are so formed are of the form $\mathrm{u} / \mathrm{rh}$ (up/ right horizontal) non-intersecting paths from $x=0$ to $x=n-1$, and ld/lh (left diagonal/ left horizontal) non-intersecting paths from $x=n+m$ to $x=n+1$ (see Figure 4).

According to (2.4) and the surrounding text the total weight of all non-intersecting $\mathrm{u} / \mathrm{rh}$ paths initially equally spaced at $y=0, \ldots,-(n-1)$ along $x=0$, finishing at $y=\mu_{j}-(j-1),(j=1, \ldots, n)$ along $x=n-1$, with up steps at $x=j-1$ weighted $b_{j}$ is given by $s_{\mu}\left(b_{1}, \ldots, b_{n}\right)$. Further, the dual paths of Figure 3 are precisely the non-intersecting $\mathrm{ld} / \mathrm{lh}$ paths initially equally spaced at $y=0, \ldots,-(n-1)$ along $x=n+m$, finishing at $y=\mu_{j}-(j-1)(j=1, \ldots, n)$ along $x=n+1$, which make up the second family in the growth process. With the possible up steps (each of unit length) at $x=n+m+1-i$ weighted by $a_{i}$, the total weight of the paths is $s_{\mu^{\prime}}\left(a_{1}, \ldots, a_{m}\right)$, where $\mu^{\prime}$ denotes the partition corresponding to the transpose of the diagram of $\mu$.

It follows from this that with an $n \times m$ array of 0's and 1's formed according to (3.1), the probability that it maps, under the dual RSK correspondence, to the above specified nested growth profile with maximum displacement $\mu$ is given by

$$
\prod_{i=1}^{m} \prod_{j=1}^{n}\left(1+a_{i} b_{j}\right)^{-1} s_{\mu^{\prime}}\left(a_{1}, \ldots, a_{m}\right) s_{\mu}\left(b_{1}, \ldots, b_{n}\right)
$$

(cf. (2.10) ). Analogous to (2.11) we have the normalization condition

$$
\prod_{i=1}^{m} \prod_{j=1}^{n}\left(1+a_{i} b_{j}\right)^{-1} \sum_{\mu} s_{\mu^{\prime}}\left(a_{1}, \ldots, a_{m}\right) s_{\mu}\left(b_{1}, \ldots, b_{n}\right)=1 .
$$


It follows immediately from (3.3) that

$$
\operatorname{Pr}\left(L_{m, n}^{01} \leq l\right)=\prod_{i=1}^{m} \prod_{j=1}^{n}\left(1+a_{i} b_{j}\right)^{-1} \sum_{\mu_{1} \leq l} s_{\mu^{\prime}}\left(a_{1}, \ldots, a_{m}\right) s_{\mu}\left(b_{1}, \ldots, b_{n}\right) .
$$

From Figure 3 and related text, we know that the Schur polynomial $s_{\mu^{\prime}}$ can be interpreted in terms of non-intersecting $\mathrm{u} / \mathrm{rh}$ paths by reading along rows, while $s_{\mu}$ can be interpreted in terms of $\mathrm{u} / \mathrm{rd}$ paths by reading down columns. In both cases the number of paths is equal to $\mu_{1}$, which according to (3.5) is no bigger than $l$. Further, the left set of paths consists of $m$ steps, and the right set consists of $n$ steps. Let $G_{n, m}^{*}\left(\vec{l}^{(0)} ; \vec{l}^{(0)}\right), \vec{l}^{(0)}:=(l-1, l-2, \ldots, 0)$ denote the total weight of such paths. Analogous to (2.12) we have that

$$
\operatorname{Pr}\left(L_{m, n}^{01} \leq l\right)=\prod_{i=1}^{m} \prod_{j=1}^{n}\left(1+a_{i} b_{j}\right)^{-1} G_{n, m}^{*}\left(\vec{l}^{(0)} ; \vec{l}^{(0)}\right) .
$$

But according to the Linström-Gessel-Viennot theorem

$$
G_{n, m}^{*}\left(\vec{l}^{(0)} ; \vec{l}^{(0)}\right)=\operatorname{det}\left[g_{n, m}^{*}\left(l_{j}^{(0)} ; l_{k}^{(0)}\right)\right]_{j, k=1, \ldots, l}
$$

where $g_{n, m}^{*}(x, y)$ is the weight of a single path of the prescribed type starting at $x$ and finishing at $y$. The latter can readily be seen to be given by

$$
g_{n, m}^{*}(x ; y)=\frac{1}{2 \pi} \int_{0}^{\pi} \prod_{j=1}^{m}\left(1+a_{j} e^{i \theta}\right) \prod_{k=1}^{n}\left(1-b_{k} e^{-i \theta}\right)^{-1} e^{-i \theta(y-x)} d \theta
$$

Substituting (3.8) in (3.7), making use of (2.15), and substituting in (3.6) we reclaim the expression for $\operatorname{Pr}\left(L_{m, n}^{01} \leq l\right)$ as a random matrix average [2],

$$
\operatorname{Pr}\left(L_{m, n}^{01} \leq l\right)=\prod_{i=1}^{m} \prod_{j=1}^{n}\left(1+a_{i} b_{j}\right)^{-1}\left\langle\left(\prod_{j=1}^{m} \prod_{k=1}^{l}\left(1+a_{j} e^{i \theta_{k}}\right)\right)\left(\prod_{j=1}^{n} \prod_{k=1}^{l}\left(1-b_{j} e^{-i \theta_{k}}\right)\right)^{-1}\right\rangle_{\mathrm{CUE}_{l}}
$$

\section{Matrices symmetric about the anti-diagonal}

With our convention of numbering rows from the bottom, the term anti-diagonal used here is what is conventionally referred to as the diagonal of the matrix. Under the RSK correspondence in the nonintersecting paths formulation, matrices symmetric about the anti-diagonal give a bijection with pairs of non-intersecting $\mathrm{u} / \mathrm{rd}$ lattice paths in which only one member of the pair is independent.

Regarding this point, reflect the entries of a general $n \times n$ non-negative integer $X$ about the antidiagonal to form the matrix $X^{R}=\left[x_{n+1-j, n+1-i}\right]_{i, j=1, \ldots, n}$. We see from the definition (2.7) that the quantities $L_{n}^{\square(l)}$ are invariant under this transformation, and thus according to (2.8) so then too are the path displacements $\mu_{l}$. Furthermore, it follows from (2.5) that

$$
\begin{aligned}
& \sum_{l=1}^{n}\left(\mu_{l}^{R}(n, j)-\mu_{l}^{R}(n, j-1)\right)=\sum_{i=1}^{n} x_{n+1-j, i}=\sum_{l=1}^{n}\left(\mu_{l}(n+1-j, n)-\mu_{l}(n-j, n)\right) \\
& \sum_{l=1}^{n}\left(\mu_{l}^{R}(i, n)-\mu_{l}^{R}(i-1, n)\right)=\sum_{i=1}^{n} x_{j, n+1-i}=\sum_{l=1}^{n}\left(\mu_{l}(n, n+1-j)-\mu_{l}(n, n-j)\right) .
\end{aligned}
$$

These equations respectively tell us that the total number of up steps at $x=-2 n-\frac{1}{2}+2 j\left(x=2 n+\frac{1}{2}-2 i\right)$

in the paths corresponding to $X^{R}$ is equal to the number of up steps at $x=2 j-\frac{3}{2}\left(x=-2 i+\frac{3}{2}\right)$ in the paths corresponding to $X$. Furthermore, if $X=X^{R}$ an algorithm can be presented (due to 
Schützenburger; see e.g. 20]) which allows paths from $x=2 n-1 / 2$ to $x=1 / 2$ to be constructed out of the paths from $x=-2 n+1 / 2$ to $x=-1 / 2$. This permits a bijection between weighted matrices symmetric about the diagonal and a single set of $\mathrm{u} / \mathrm{rh}$ lattice paths provided the weighting of $x_{i, j}$ in the former is equal to $\left(a_{i} a_{n+1-j}\right)^{x_{i, j}}$ (and thus $b_{n+1-i}=a_{i}$ ). For example, the matrix of Figure 2 is symmetric about the anti-diagonal. With $n=2$, setting $b_{n+1-i}=a_{i}(i=1, \ldots, n)$ we see that the weight of steps at $x=-2 n-1 / 2+2 i$ is equal to the weight of up steps at $x=2 i-1 / 2(i=1, \ldots, n)$.

With the constraint $X=X^{R}$, to obtain a probabilistic setting we should weight only the sites $i \leq n+1-j$. To achieve this without affecting the weights of the pairs of paths we simply square the weights at the sites $i<n+1-j$, and set the weights at sites $i>n+1-j$ to unity. With $a_{i}=\sqrt{q_{i}}$, we therefore choose

$$
\begin{aligned}
& \operatorname{Pr}\left(x_{i, j}=k\right)=\left(1-q_{i} q_{n+1-j}\right)\left(q_{i} q_{n+1-j}\right)^{k}, \quad i<n+1-j, \\
& \operatorname{Pr}\left(x_{i, n+1-i}=k\right)=\left(1-q_{i}\right) q_{i}^{k}
\end{aligned}
$$

which corresponds to weighting the vertical segments of the single $\mathrm{u} / \mathrm{rh}$ paths by $q_{1}, q_{2}, \ldots, q_{n}$ from right to left. According to (2.4) with the maximum displacement of the level- $l$ path denoted by $\mu_{l}$, the total weight of such paths is given by $s_{\mu}\left(q_{1}, \ldots, q_{n}\right)$. We conclude that under the RSK mapping, with the independent entries chosen according to (4.2), the probability a non-negative integer matrix symmetric about the anti-diagonal maps to a pair of $\mathrm{u} / \mathrm{rh}$ paths with final displacement $\mu$ is equal to

$$
\prod_{i=1}^{n}\left(1-q_{i}\right) \prod_{1 \leq i<j \leq n}\left(1-q_{i} q_{j}\right) s_{\mu}\left(q_{1}, \ldots, q_{n}\right) .
$$

The specification (4.2) can be generalized, allowing for a generalization of (4.3). For this one recalls [15] that the RSK correspondence has the property that for $\left[x_{i, j}\right]$ symmetric about the anti-diagonal

$$
\#\left\{x_{i, n+1-i}: x_{i, n+1-i} \text { odd }\right\}=\#\left\{\mu_{j}: \mu_{j} \text { odd }\right\}=\sum_{j=1}^{n}(-1)^{j-1} \mu_{j}^{\prime}
$$

where $\mu_{j}^{\prime}$ denotes the displacement of the level $j$ conjugate path or equivalently the length of the $j$ th column in the corresponding tableau (recall Figure 3). Hence if we generalize the second probability in (4.2) to read

$$
\operatorname{Pr}\left(x_{i, n+1-i}=k\right)=\frac{\left(1-q_{i}^{2}\right)}{1+\beta q_{i}} \beta^{k \bmod 2} q_{i}^{k}
$$

then we have that (4.3) generalizes to

$$
\prod_{i=1}^{n} \frac{\left(1-q_{i}^{2}\right)}{1+\beta q_{i}} \prod_{1 \leq i<j \leq n}\left(1-q_{i} q_{j}\right) \beta^{\sum_{j=1}^{n}(-1)^{j-1} \mu_{j}^{\prime}} s_{\mu}\left(q_{1}, \ldots, q_{n}\right) .
$$

Writing

$$
L_{n}^{\bowtie}:=\max \sum_{\substack{(1,1) \mathrm{u} / \mathrm{rh}(n, n) \\ X=X^{R}}} x_{i, j}
$$

it follows from this that 2

$$
\operatorname{Pr}\left(L_{n}^{\triangleright} \leq l\right)=\prod_{i=1}^{n} \frac{1-q_{i}^{2}}{1+\beta q_{i}} \prod_{1 \leq i<j \leq n}\left(1-q_{i} q_{j}\right) \sum_{\mu: \mu_{1} \leq l} \beta^{\sum_{j=1}^{n}(-1)^{j-1} \mu_{j}^{\prime}} s_{\mu}\left(q_{1}, \ldots, q_{n}\right) .
$$

Using symmetric function theory, Baik and Rains [2] show that the sum in (4.6) can be written as a random matrix average involving the classical group $S p(2 l)$. Matrices from this subgroup of $U(2 l)$ have their eigenvalues in complex conjugate pairs $\left\{e^{ \pm i \theta_{j}}\right\}_{j=1, \ldots, l}$, with $0<\theta_{j}<\pi(j=1, \ldots, l)$. Here we will give a derivation in keeping with integration techniques from random matrix theory. 
Proposition 1. Consider the eigenvalues with angles $0<\theta_{j}<\pi(j=1, \ldots, l)$ of matrices from $S p(2 l)$. Let \langle\rangle$_{S p(2 l)}$ denote an average with respect to the corresponding eigenvalue p.d.f.,

$$
\frac{1}{(2 \pi)^{l}} \frac{1}{2^{l} l !} \prod_{k=1}^{l}\left|e^{i \theta_{k}}-e^{-i \theta_{k}}\right|^{2} \prod_{1 \leq j<k \leq l}\left|e^{i \theta_{j}}-e^{i \theta_{k}}\right|^{2}\left|1-e^{i\left(\theta_{j}+\theta_{k}\right)}\right|^{2} .
$$

One has

$$
\begin{aligned}
& \operatorname{Pr}\left(L_{n}^{\triangleright} \leq 2 l\right)=\prod_{i=1}^{n} \frac{1-q_{i}^{2}}{1+\beta q_{i}} \prod_{1 \leq i<j \leq n}\left(1-q_{i} q_{j}\right)\left\langle\prod_{k=1}^{l}\left(\frac{1}{\left|1-\beta e^{-i \theta_{k}}\right|^{2}} \prod_{j=1}^{n}\left|1+q_{j} e^{i \theta_{k}}\right|^{2}\right)\right\rangle_{S p(2 l)} \\
& \operatorname{Pr}\left(L_{n}^{\square} \leq 2 l+1\right)=\prod_{i=1}^{n}\left(1-q_{i}^{2}\right) \prod_{1 \leq i<j \leq n}\left(1-q_{i} q_{n+1-j}\right)\left\langle\prod_{k=1}^{l} \prod_{j=1}^{n}\left|1+q_{j} e^{i \theta_{k}}\right|^{2}\right\rangle_{S p(2 l)} .
\end{aligned}
$$

Proof The maximum possible height in the growth model relating to (3.5) is $m$, implying the so called dual Cauchy identity

$$
\prod_{i=1}^{m} \prod_{j=1}^{n}\left(1+a_{i} b_{j}\right)=\sum_{\mu_{1} \leq m} s_{\mu^{\prime}}\left(a_{1}, \ldots, a_{m}\right) s_{\mu}\left(b_{1}, \ldots, b_{n}\right) .
$$

Renaming the parameters, it follows from this that

$$
\prod_{k=1}^{l} \prod_{j=1}^{n}\left|1+q_{j} e^{i \theta_{k}}\right|^{2}=\sum_{\mu: \mu_{1} \leq 2 l} s_{\mu}\left(q_{1}, \ldots, q_{n}\right) s_{\mu^{\prime}}\left(e^{i \theta_{1}}, e^{-i \theta_{1}}, \ldots, e^{i \theta_{l}}, e^{-i \theta_{l}}\right) .
$$

Substituting (4.11) in (4.8), substituting the result in (4.6), equating coefficients of $s_{\mu}\left(q_{1}, \ldots, q_{n}\right)$ and writing $\mu^{\prime}=\rho$ shows that (4.8) is equivalent to the matrix integral formula

$$
\left\langle\prod_{k=1}^{l} \frac{1}{\left|1-\beta e^{-i \theta_{k}}\right|^{2}} s_{\rho}\left(e^{i \theta_{1}}, e^{-i \theta_{1}}, \ldots, e^{i \theta_{l}}, e^{-i \theta_{l}}\right)\right\rangle_{S p(2 l)}=\beta^{\sum_{j=1}^{2 l}(-1)^{j-1} \rho_{j}} .
$$

Now, the meaning of the matrix integral is an integral over $\theta_{k} \in[0, \pi]$, weighted by (4.7). Noting that the integrand is unchanged by $\theta_{l} \mapsto-\theta_{l}$, and making use of the determinant formula for Schur polynomials

$$
s_{\lambda}\left(x_{1}, \ldots, x_{N}\right)=\frac{\operatorname{det}\left[q_{j}^{N-k+\lambda_{k}}\right]_{j, k=1, \ldots, N}}{\operatorname{det}\left[q_{j}^{N-k}\right]_{j, k=1, \ldots, N}} .
$$

shows that the matrix integral is equal to

$$
\frac{1}{(2 \pi)^{l} 2^{l} l !} \int_{-\pi}^{\pi} d \theta_{1} \cdots \int_{-\pi}^{\pi} d \theta_{l} \prod_{k=1}^{l} \frac{\left(e^{i \theta_{k}}-e^{-i \theta_{k}}\right)}{\left|1-\beta e^{-i \theta_{k}}\right|^{2}} \operatorname{det}\left[\begin{array}{l}
e^{i \theta_{j}\left(\rho_{2 l-k+1}+k-1\right)} \\
e^{-i \theta_{j}\left(\rho_{2 l-k+1}+k-1\right)}
\end{array}\right]_{\substack{j=1, \ldots, l \\
k=1, \ldots, 2 l}} .
$$

The structure of the integral (4.14) is very common in random matrix theory [18, 6]. It can be written as a Pfaffian, giving that (4.14) is equal to

$$
\frac{1}{2^{l}} \operatorname{Pf}\left[\frac{1}{2 \pi} \int_{-\pi}^{\pi} \frac{e^{i \theta}-e^{-i \theta}}{\left|1-\beta e^{-i \theta}\right|^{2}}\left(e^{i \theta\left(\rho_{j}-j-\rho_{k}+k\right)}-e^{-i \theta\left(\rho_{j}-j-\rho_{k}+k\right)}\right) d \theta\right]_{j, k=1, \ldots, 2 l},
$$

which after evaluating the integral therein reduces to

$$
\beta^{-l} \operatorname{Pf}\left[\operatorname{sgn}(k-j) \beta^{\left|\rho_{j}-\rho_{k}+k-j\right|}\right]_{j, k=1, \ldots, 2 l} \cdot
$$


This Pfaffian is special case $x_{j}=\rho_{j}-j, f\left(x_{j}\right)=\beta^{x_{j}}$ in the general formula 8

$$
\operatorname{Pf}\left[\left(\frac{f\left(x_{j}\right)}{f\left(x_{k}\right)}\right)^{\operatorname{sgn}\left(x_{j}-x_{k}\right)} \operatorname{sgn}\left(x_{j}-x_{k}\right)\right]_{j, k=1, \ldots, 2 l}=\prod_{j=1}^{l} \frac{f\left(x_{Q(2 j-1)}\right)}{f\left(x_{Q(2 j)}\right)} \varepsilon(Q),
$$

where the permutation $Q$ is such that

$$
x_{Q(2 j-1)}>x_{Q(2 j)}, \quad Q(2 j)>Q(2 j-1)(j=1, \ldots, l)
$$

and thus evaluating to the r.h.s. of (4.12).

In regards to (4.9), use of an appropriate modification of (4.11) shows that this is equivalent to the matrix integral formula

$$
\left\langle s_{\rho}\left(e^{i \theta_{1}}, e^{-i \theta_{1}}, \ldots, e^{i \theta_{l}}, e^{-i \theta_{l}}, \beta\right)\right\rangle_{S p(2 l)}=\beta^{\sum_{j=1}^{2 l+1}(-1)^{j-1} \rho_{j}} .
$$

To derive this, use of (4.7) and (4.13) shows that the matrix integral is equal to

$$
\begin{aligned}
& \frac{1}{(2 \pi)^{l} 2^{l} l !} \int_{-\pi}^{\pi} d \theta_{1} \cdots \int_{-\pi}^{\pi} d \theta_{l} \prod_{k=1}^{l} \frac{\left(e^{i \theta_{k}}-e^{-i \theta_{k}}\right)}{\mid 1-\beta e^{-\left.i \theta_{k}\right|^{2}}} \operatorname{det}\left[\begin{array}{c}
e^{i \theta_{j}\left(\rho_{2 l-k+2}+k-1\right)} \\
e^{-i \theta_{j}\left(\rho_{2 l-k+2}+k-1\right)} \\
\beta^{\rho_{2 l-k+2}+k-1}
\end{array}\right]_{\substack{j=1, \ldots, l \\
k=1, \ldots, 2 l+1}} \\
& =\frac{1}{2^{l}} \operatorname{Pf}\left[\begin{array}{cc}
A_{(2 l+1) \times(2 l+1)} & {\left[\beta^{\rho_{j}+2 l+1-j}\right]_{j=1, \ldots, 2 l+1}} \\
{\left[-\beta^{\rho_{k}+2 l+1-k}\right]_{k=1, \ldots, 2 l+1}} & 0
\end{array}\right] .
\end{aligned}
$$

where

$$
A_{(2 l+1) \times(2 l+1)}:=\left[\frac{1}{2 \pi} \int_{-\pi}^{\pi} \frac{e^{i \theta}-e^{-i \theta}}{\left|1-\beta e^{-i \theta}\right|^{2}}\left(e^{i \theta\left(\rho_{j}-j-\rho_{k}+k\right)}-e^{-i \theta\left(\rho_{j}-j-\rho_{k}+k\right)}\right) d \theta\right]_{j, k=1, \ldots, 2 l+1} .
$$

Here the second equality follows from standard integration methods of random matrix theory, in the same way that (4.15) follows from (4.14). Computing the integral reduces this to

$$
\beta^{-(l+1)} \operatorname{Pf}\left[\begin{array}{cc}
{\left[\operatorname{sgn}(k-j) \beta^{\left|\rho_{j}-\rho_{k}+k-j\right|}\right]_{j, k=1, \ldots, 2 l+1}} & {\left[\beta^{\rho_{j}+2 l+1-j}\right]_{j=1, \ldots, 2 l}} \\
{\left[-\beta^{\rho_{k}+2 l+1-k}\right]_{k=1, \ldots, 2 l}} & 0
\end{array}\right] .
$$

But this is precisely the same as (4.16) with $l \mapsto l+1, \rho_{2 l+2}=0$, and so reduces to the r.h.s. of (4.18).

\section{Matrices symmetric about the diagonal}

According to the rules of the polynuclear growth model, if a non-negative integer matrix $X=\left[x_{i, j}\right]_{i, j=1, \ldots, n}$ maps to a pair of $\mathrm{u} / \mathrm{rh}$ non-intersecting lattice paths $\left(P_{1}, P_{2}\right)$ of the same final displacement, then the transposed matrix $X^{T}=\left[x_{j, i}\right]_{i, j=1, \ldots, n}$ maps to the pair of $\mathrm{u} / \mathrm{rh}$ non-intersecting paths $\left(P_{2}, P_{1}\right)$. Hence the Robinson-Schensted-Knuth correspondence when applied to symmetric matrices $X=X^{T}$ gives a bijection with a single set of $\mathrm{u} / \mathrm{rh}$ lattice paths, since then we must have $P_{1}=P_{2}$. To obtain a bijection between weighted symmetric matrices and a weighted set of $\mathrm{u} / \mathrm{rh}$ lattice paths, and furthermore to obtain a probabilistic setting, we weight only the entries $i \leq j$, with the value of $x_{i, j}$ for $i>j$ fixed by symmetry. Arguing then as in the derivation of (4.3) we see with

$$
\operatorname{Pr}\left(x_{i, j}=k\right)=\left(1-q_{i} q_{j}\right)\left(q_{i} q_{j}\right)^{k}, i<j \quad \operatorname{Pr}\left(x_{i, i}=k\right)=\left(1-q_{i}\right) q_{i}^{k}
$$


the probability the symmetric matrix $X$ maps to a set of $\mathrm{u} / \mathrm{rh}$ paths with final displacements $\mu$ is equal to $[13$ ]

$$
\prod_{i=1}^{n}\left(1-q_{i}\right) \prod_{1 \leq i<j \leq n}\left(1-q_{i} q_{j}\right) s_{\mu}\left(q_{1}, \ldots, q_{n}\right) .
$$

As with (4.3) this can be generalized to the case that the diagonal entries are chosen according to

$$
\operatorname{Pr}\left(x_{i, i}=k\right)=\left(1-\alpha q_{i}\right)\left(\alpha q_{i}\right)^{k} .
$$

Thus recalling [15, 9] that in the Robinson-Schensted-Knuth correspondence for symmetric matrices

$$
\sum_{j=1}^{n} x_{j, j}=\sum_{j=1}^{n}(-1)^{j-1} \mu_{j}
$$

with the generalization (5.3), (5.2) should correspondingly be generalized to read [2]

$$
\prod_{i=1}^{n}\left(1-\alpha q_{i}\right) \prod_{1 \leq i<j \leq n}\left(1-q_{i} q_{j}\right) \alpha^{\sum_{j=1}^{n}(-1)^{j-1} \mu_{j}} s_{\mu}\left(q_{1}, \ldots, q_{n}\right) .
$$

Writing

$$
L_{n}^{\square}:=\max \sum_{\substack{(1,1) \mathrm{u} / \mathrm{rh}(n, n) \\ X=X^{T}}} x_{i, j}
$$

and noting that

$$
\sum_{j=1}^{n}(-1)^{j-1} \mu_{j}=\#(\text { columns of odd length in } \mu)=\sum_{k=1}^{l} \mu_{k}^{\prime} \bmod 2
$$

where $l=\mu_{1}$, it follows from (5.5) that

$$
\operatorname{Pr}\left(L_{n}^{\square} \leq l\right)=\prod_{i=1}^{n}\left(1-\alpha q_{i}\right) \prod_{1 \leq i<j \leq n}\left(1-q_{i} q_{j}\right) \sum_{\mu: \mu_{1} \leq l} \alpha^{\sum_{k=1}^{l} \mu_{k}^{\prime} \bmod 2} s_{\mu}\left(q_{1}, \ldots, q_{n}\right) .
$$

Starting with this formula, Baik and Rains [2] proved the following analogue of Proposition 1, involving now a random matrix average involving the classical group $O(l)$. Matrices from this subgroup of $U(l)$ form two disjoint components, $O^{+}(l)$ and $O^{-}(l)$, distinguished by the determinant equalling +1 or -1 respectively. The complex eigenvalues occur in complex conjugate pairs, and there is a real eigenvalue $z=-1$ for matrices in $O^{-}(l)$ with $l$ odd, a real eigenvalue eigenvalue $z=1$ for matrices in $O^{+}(l)$ with $l$ even, and two real eigenvalues $z= \pm 1$ for matrices in $O^{-}(l)$ with $l$ even.

Proposition 2. Consider the eigenvalues with angles $0<\theta_{j}<\pi,(j=1, \ldots, l)$ of matrices from $O(l)$. Define

$$
\langle\cdot\rangle_{O(l)}=\frac{1}{2}\left(\langle\cdot\rangle_{O^{+}(l)}+\langle\cdot\rangle_{O^{-}(l)}\right)
$$

where $\langle\cdot\rangle_{O^{+}(l)}$ denotes an average with respect to the eigenvalue p.d.f. for random matrices from the classical group $\mathrm{O}^{+}(l)$,

$$
\begin{aligned}
& \frac{1}{\pi^{l / 2} 2^{l-1}(l / 2) !} \prod_{1 \leq j<k \leq l / 2}\left|e^{i \theta_{j}}-e^{i \theta_{k}}\right|^{2}\left|1-e^{i\left(\theta_{j}+\theta_{k}\right)}\right|^{2}, \quad l \text { even } \\
& \frac{1}{\pi^{(l-1) / 2} 2^{l-1}((l-1) / 2) !} \prod_{j=1}^{(l-1) / 2}\left|1-e^{i \theta_{j}}\right|^{2} \prod_{1 \leq j<k \leq(l-1) / 2}\left|e^{i \theta_{j}}-e^{i \theta_{k}}\right|^{2}\left|1-e^{i\left(\theta_{j}+\theta_{k}\right)}\right|^{2}, \quad l \text { odd, }
\end{aligned}
$$


and $\langle\cdot\rangle_{O^{-}(l)}$ denotes an average with respect to the eigenvalue p.d.f. for random matrices from the classical group $O^{-}(l)$,

$$
\begin{aligned}
& \frac{1}{\pi^{l / 2-1} 2^{l-2}(l / 2) !} \prod_{k=1}^{l / 2-1}\left|1-e^{2 i \theta_{k}}\right|^{2} \prod_{1 \leq j<k \leq l / 2-1}\left|e^{i \theta_{j}}-e^{i \theta_{k}}\right|^{2}\left|1-e^{i\left(\theta_{j}+\theta_{k}\right)}\right|^{2}, \quad l \text { even } \\
& \frac{1}{\pi^{(l-1) / 2} 2^{l-1}((l-1) / 2) !} \delta\left(\theta_{l}-\pi\right) \prod_{j=1}^{(l-1) / 2}\left|1+e^{i \theta_{j}}\right|^{2} \prod_{1 \leq j<k \leq(l-1) / 2}\left|e^{i \theta_{j}}-e^{i \theta_{k}}\right|^{2}\left|1-e^{i\left(\theta_{j}+\theta_{k}\right)}\right|^{2}
\end{aligned}
$$

We have

$$
\operatorname{Pr}\left(L_{n}^{\square} \leq l\right)=\prod_{i=1}^{n}\left(1-\alpha q_{i}\right) \prod_{1 \leq i<j \leq n}\left(1-q_{i} q_{j}\right)\left\langle\operatorname{det}\left(\mathbf{1}_{l}+\alpha U\right) \prod_{j=1}^{n}\left(\mathbf{1}_{l}+q_{j} U\right)\right\rangle_{U \in \mathrm{O}(l)} .
$$

Proof. Use of the dual Cauchy identity (4.10) in (5.11) and comparison with (5.6) shows that (5.11) is equivalent to the matrix integral evaluation

$$
\left\langle\operatorname{det}\left(\mathbf{1}_{l}+\alpha U\right) s_{\rho}(U)\right\rangle_{U \in O(l)}=\alpha^{\sum_{j=1}^{l} \rho_{j} \bmod 2}
$$

where $s_{\rho}(U)$ denotes the Schur polynomial as a function of all the eigenvalues of $U$.

Consider first the $l$ even case, $l \mapsto 2 l$, and consider separately the components $O^{ \pm}(2 l)$ of $O(2 l)$. Substituting the eigenvalue p.d.f. for $O^{+}(2 l)$ (5.7), and proceeding as in the derivation of (4.16), which involves use of (4.13) and reduction to a Pfaffian, shows

$$
\left\langle\operatorname{det}\left(\mathbf{1}_{2 l}+\alpha U\right) s_{\rho}(U)\right\rangle_{U \in O^{+}(2 l)}=2^{1-l} \operatorname{Pf}\left[a_{j k}\right]_{j, k=1, \ldots, 2 l}
$$

where

$$
\begin{aligned}
a_{j k} & =\left(\left(1+\alpha^{2}\right) \delta_{\left(\rho_{j}-j\right)-\left(\rho_{k}-k\right), \text { odd }}+2 \alpha \delta_{\left(\rho_{j}-j\right)-\left(\rho_{k}-k\right), \text { even }}\right) \operatorname{sgn}(k-j) \\
& =\left(\frac{1}{2}(1+\alpha)^{2}-\frac{1}{2}(1-\alpha)^{2}(-1)^{\left(\rho_{j}-j\right)-\left(\rho_{k}-k\right)}\right) \operatorname{sgn}(k-j)
\end{aligned}
$$

The task is therefore to compute the Pfaffian of the matrix with these entries. For this one uses the identity 22

$$
\operatorname{Pf}(A+B)=\sum_{\substack{S \subseteq\{1,2, \ldots, 2 l\} \\|S| \text { even }}}(-1)^{\sum_{j \in S} j-|S| / 2} \operatorname{Pf}_{S}(A) \operatorname{Pf}_{\bar{S}}(B)
$$

where $\operatorname{Pf}_{S}(A)$ denotes the Pfaffian of $A$ restricted to rows and columns specified by the index set $S$, and similarly $\operatorname{Pf}_{\bar{S}}(B)$. With

$$
A=\left[\frac{1}{2}(1+\alpha)^{2} \operatorname{sgn}(k-j)\right]_{j, k=1, \ldots, 2 l}, \quad B=\left[-\frac{1}{2}(1-\alpha)^{2}(-1)^{\left(\rho_{j}-j\right)-\left(\rho_{k}-k\right)} \operatorname{sgn}(k-j)\right]_{j, k=1, \ldots, 2 l},
$$

and noting

$$
\operatorname{Pf}[\operatorname{sgn}(k-j)]=1, \quad \operatorname{Pf}\left[a_{j, k}(-1)^{\left(\rho_{j}-j\right)-\left(\rho_{k}-k\right)}\right]=(-1)^{\sum\left(\rho_{j}-j\right)} \operatorname{Pf}\left[a_{j, k}\right]
$$

we see that

$$
\operatorname{Pf}_{S} A=2^{-|S| / 2}(1+\alpha)^{|S|}, \quad \operatorname{Pf}_{\bar{S}} B=(-2)^{-|\bar{S}| / 2}(1-\alpha)^{2 l-|S|}(-1)^{\sum_{j \in \bar{S}} \rho_{j}-j}
$$


It thus follows from (5.15) that

$$
2^{1-l} \operatorname{Pf}(A+B)=2 \sum_{\substack{S \subseteq\{1,2, \ldots, 2 l\} \\|S| \text { even }}}\left(\frac{1+\alpha}{2}\right)^{|S|}\left(\frac{1-\alpha}{2}\right)^{2 l-|S|}(-1)^{\sum_{j \in \bar{S}} \rho_{j}} .
$$

Now, in general

$$
\begin{aligned}
& \sum_{\substack{S \subseteq\{1,2, \ldots, 2 l\} \\
|S| \text { even }}} x^{|S|} y^{2 l-|S|}(-1)^{\sum_{j \in \bar{S}} \rho_{j}} \\
& \quad=\frac{1}{2}\left(\sum_{S \subseteq\{1,2, \ldots, 2 l\}} x^{|S|} y^{2 l-|S|}(-1)^{\sum_{j \in \bar{S}} \rho_{j}}+\sum_{S \subseteq\{1,2, \ldots, 2 l\}} x^{|S|}(-y)^{2 l-|S|}(-1)^{\sum_{j \in \bar{S}} \rho_{j}}\right) \\
& \quad=\frac{1}{2}\left(\prod_{j=1}^{2 l}\left(x+(-1)^{\rho_{j}} y\right)+\prod_{j=1}^{2 l}\left(x-(-1)^{\rho_{j}} y\right)\right) .
\end{aligned}
$$

Using this result to evaluate (5.16) and substituting in (5.13) gives the matrix integral evaluation

$$
\left\langle\operatorname{det}\left(\mathbf{1}_{2 l}+\alpha U\right) s_{\rho}(U)\right\rangle_{U \in O^{+}(2 l)}=\alpha^{\sum_{j=1}^{2 l} \rho_{j} \bmod 2}+\alpha^{\sum_{j=1}^{2 l}\left(\rho_{j}+1\right) \bmod 2} .
$$

We turn now to the corresponding formula for the average over $O^{-}(2 l)$. The analogue of (5.13) in this case is

$$
\left\langle\operatorname{det}\left(\mathbf{1}_{2 l}+\alpha U\right) s_{\rho}(U)\right\rangle_{U \in O^{-}(2 l)}=\frac{\left(1-\alpha^{2}\right)}{2^{l-1}}[\zeta] \operatorname{Pf}\left[a_{j, k}+\zeta b_{j, k}\right]_{j, k=1, \ldots, 2 l}
$$

where $a_{j, k}$ is as in (5.13) while $b_{j, k}=(-1)^{\rho_{k}-k}-(-1)^{\rho_{j}-j}$, and [ $\left.\zeta\right]$ denotes the coefficient of $\zeta$. Observing that

$$
\left[b_{j k}\right]=\vec{u} \vec{w}^{T}-\vec{w} \vec{u}^{T}, \quad \vec{u}=[1]_{j=1, \ldots, 2 l}, \quad \vec{w}=\left[(-1)^{\rho_{j}-j}\right]_{j=1, \ldots, 2 l}
$$

shows that $\left[b_{j k}\right]$ has rank 2. It follows that the Pfaffian in (5.18) is linear in $\zeta$, and so the r.h.s. of (5.18) can be rewritten

$$
\frac{\left(1-\alpha^{2}\right)}{2^{l-1}} \frac{1}{\zeta}\left(\operatorname{Pf}\left[\left[a_{j k}\right]+\zeta\left[b_{j k}\right]\right]-\operatorname{Pf}\left[a_{j k}\right]\right) .
$$

With $\gamma, \zeta_{1}, \zeta_{2}$ arbitrary non-zero constants, the structure of $\left[b_{j k}\right]$ and use of elementary row and column operations verifies that this in turn can be rewritten

$$
\frac{\left(1-\alpha^{2}\right)}{2^{l-1}} \frac{1}{\zeta_{1} \zeta_{2}}\left(\operatorname{Pf}\left[\begin{array}{ccc}
{\left[a_{j k}\right]} & \zeta_{1} \vec{w} & \zeta_{2} \vec{u} \\
-\zeta_{1} \vec{w}^{T} & 0 & \gamma \\
-\zeta_{2} \vec{u}^{T} & -\gamma & 0
\end{array}\right]-\gamma \operatorname{Pf}\left[a_{j k}\right]\right) .
$$

Setting $\zeta_{1}=\frac{1}{2}(1-\alpha)^{2}, \quad \zeta_{2}=(1+\alpha)^{2}$, adding one half of the final row/column to the second last row/column, and subtracting the second last row/column from the final row column, then setting $\gamma=\left(1+\alpha^{2}\right)$ allows (5.21) to be rewritten as

$$
\frac{2^{1-l}}{1-\alpha^{2}}\left(\left.\operatorname{Pf}\left[a_{j k}\right]_{2(l+1) \times 2(l+1)}\right|_{\rho_{2 l+1}=\rho_{2 l+2}=0}-\left(1+\alpha^{2}\right) \operatorname{Pf}\left[a_{j k}\right]_{2 l \times 2 l}\right) .
$$

Comparing (5.13) and (5.17) tells us that

$$
\operatorname{Pf}\left[a_{j k}\right]_{2 l \times 2 l}=2^{l-1}\left(\alpha^{\sum_{j=1}^{2 l} \rho_{j} \bmod 2}+\alpha^{\sum_{j=1}^{2 l}\left(\rho_{j}+1\right) \bmod 2}\right) .
$$

Substituting in (5.22) and simplifying implies the matrix integral evaluation

$$
\left\langle\operatorname{det}\left(\mathbf{1}_{2 l}+\alpha U\right) s_{\rho}(U)\right\rangle_{U \in O^{-}(2 l)}=\alpha^{\sum_{j=1}^{2 l} \rho_{j} \bmod 2}-\alpha^{\sum_{j=1}^{2 l}\left(\rho_{j}+1\right) \bmod 2} .
$$


Adding this to (5.17) verifies (5.12) in the case $l$ even.

Similar working suffices in the $l$ odd case, $l \mapsto 2 l+1$. In regards to the average over $O^{+}(2 l+1)$, making use of the explicit form of the p.d.f. (5.9), the determinant form of the Schur polynomial (4.13), and integration techniques from random matrix theory, one obtains the Pfaffian formula

$$
\left\langle\operatorname{det}\left(\mathbf{1}_{2 l+1}+\alpha U\right) s_{\rho}(U)\right\rangle_{U \in O^{+}(2 l+1)}=\frac{(1+\alpha)}{2^{l}} \operatorname{Pf}\left[\begin{array}{cc}
{\left[a_{j k}\right]_{j, k=1, \ldots, 2 l+1}} & {[1]_{j=1, \ldots, 2 l+1}} \\
-[1]_{k=1, \ldots, 2 l+1} & 0
\end{array}\right]
$$

where $a_{j k}$ is specified by (5.14). This Pfaffian can in fact be evaluated by making use of the Pfaffian evaluation implied by the equality of (5.13) and (5.17). To see this, multiply the final row and column of the matrix in (5.24) by $(1+\alpha)^{2}$, and balance the equation by dividing by a prrefactor of $(1+\alpha)^{2}$ on the r.h.s.. Next subtract the 2nd last row from the final row, and 2nd last column from the final column. Finally, write in the 2 nd last entry of the final row and column $(1+\alpha)^{2}=\left(1+\alpha^{2}\right)+2 \alpha$. This shows that (5.24) is equal to

$$
\frac{1}{2^{l}(1+\alpha)} \operatorname{Pf}\left[\left.A_{(2 l+2) \times(2 l+2)}\right|_{\rho_{2 l+2}=\rho_{2 l+1}}+B^{\prime}\right]
$$

where $A_{(2 l+2) \times(2 l+2)}:=\left[a_{j k}\right]_{j, k=1, \ldots, 2 l+2}$ while $B^{\prime}$ has all entries zero except for the second last entry of the final column, which is $2 \alpha$, and the second last entry of the final row, which is $-2 \alpha$. Making use of (5.15) shows that this in turn is equal to

$$
\frac{1}{2^{l}(1+\alpha)}\left(\left.\operatorname{Pf} A_{(2 l+2) \times(2 l+2)}\right|_{\rho_{2 l+2}=\rho_{2 l+1}}+2 \alpha \operatorname{Pf} A_{2 l \times 2 l}\right) .
$$

But the value of $\operatorname{Pf} A_{2 l \times 2 l}$ for general $l$ is known from the equality between (5.13) and (5.17), so we find that (5.27) reduces to

$$
\alpha^{\sum_{j=1}^{2 l+1} \rho_{j} \bmod 2}+\alpha^{\sum_{j=1}^{2 l+1}\left(\rho_{j}+1\right) \bmod 2}
$$

thus giving the evaluation of the random matrix average in (5.24).

For the average over $O^{-}(2 l+1)$, we note that a change of variables $\theta_{j} \mapsto \pi-\operatorname{theta}_{j}(j=1, \ldots, l)$ shows

$$
\left\langle\operatorname{det}\left(\mathbf{1}_{2 l+1}+\alpha U\right) s_{\rho}(U)\right\rangle_{U \in O^{-}(2 l+1)}=(-1)^{|\rho|}\left\langle\operatorname{det}\left(\mathbf{1}_{2 l+1}-\alpha U\right) s_{\rho}(U)\right\rangle_{U \in O^{+}(2 l+1)} .
$$

Substituting (5.27) with $\alpha \mapsto-\alpha$ for the average on the r.h.s. shows that this is equal to

$$
\alpha^{\sum_{j=1}^{2 l+1} \rho_{j} \bmod 2}-\alpha^{\sum_{j=1}^{2 l+1}\left(\rho_{j}+1\right) \bmod 2} .
$$

Finally, taking the arithmetic mean of (5.27) and (5.29), we obtain the sought evaluation (5.12) with $l \mapsto 2 l+1$.

\section{Matrices symmetric about both the diagonal and anti-diagonal}

Let the $2 n \times 2 n$ matrix $X=\left[x_{i, j}\right]_{i, j=1, \ldots, 2 n}$ have non-negative integer entries, and label the rows from the bottom. Suppose furthermore the entries are symmetric with respect to reflections in both the diagonal $\left(x_{i, j}=x_{j, i}, i>j\right)$ and anti-diagonal $\left(x_{i, j}=x_{i, 2 n+1-j}, i>2 n+1-j\right)$. Because $X$ is symmetric about the diagonal, the RSK correspondence maps $X$ to a pair of identical non-intersecting u/rh lattice paths $(P, P)$ say. On the other hand $X$ being symmetric about the anti-diagonal implies $X$ maps to the lattice path pair $\left(P^{R}, P\right)$, where $P^{R}$ is the Schützenberger dual of $P$. Consequently in this case $X$ maps under

the RSK correspondence to a single set of at most $2 n \mathrm{u} / \mathrm{rh}$ non-intersecting lattice paths $P$ with the special property that $P=P^{R}$. 
We will suppose furthermore that the entries on the anti-diagonal are constrained to be even. Then according to (4.4) all final displacements $\mu_{i}$ of the paths must be even. A partition with parts $2 \lambda_{i}$ so each part is even will be written $2 \lambda$.

The independent elements of $X$ can be chosen to be $x_{i, j}$ with $i \leq j(i, j=1, \ldots, n)$ and $i \leq 2 n+1-j$ $(i=1, \ldots, n, j=n+1, \ldots, 2 n)$. We choose the value of each such $x_{i, j}$, excluding those on the diagonal or anti-diagonal, according to the geometric distribution

$$
\operatorname{Pr}\left(x_{i, j}=k\right)=\left(1-q_{i} q_{j}\right)\left(q_{i} q_{j}\right)^{k}
$$

On the anti-diagonal we choose

$$
\operatorname{Pr}\left(x_{i, 2 n+1-i}=k\right)= \begin{cases}\left(1-q_{i}^{2}\right) q_{i}^{k}, & k \text { even } \\ 0, & \text { otherwise }\end{cases}
$$

and on the diagonal

$$
\operatorname{Pr}\left(x_{i, i}=k\right)=\left(1-\alpha q_{i}\right)\left(\alpha q_{i}\right)^{k} \quad(i=1, \ldots, n) .
$$

The bijection then implies that the probability $X$ maps to a set of at most $2 n$ non-intersecting $\mathrm{u} / \mathrm{rh}$ lattice paths of final displacement $2 \lambda$ is equal to

$$
\prod_{i=1}^{n}\left(\left(1-q_{i}^{2}\right)\left(1-\alpha q_{i}\right) \prod_{j=i+1}^{n}\left(1-q_{i} q_{j}\right) \prod_{j=n+1}^{n+i}\left(1-q_{i} q_{j}\right)\right) \alpha^{\sum_{l=1}^{2 n} \lambda_{l}} s_{2 \lambda}^{\text {s.d. }}\left(q_{1}, \ldots, q_{2 n}\right)
$$

where

$$
s_{2 \lambda}^{\text {s.d. }}\left(q_{1}, \ldots, q_{2 n}\right):=\sum^{*} \prod_{j=1}^{2 n} q_{j}^{\frac{1}{2} \sum_{l=1}^{2 n} \tilde{\lambda}_{l}(j)}
$$

with $\tilde{\lambda}_{l}(j)$ denoting the number of vertical steps at $x=j-1$ contained in the level- $l$ path and the asterisk denoting that the sum is over all self dual $\mathrm{u} / \mathrm{rh}$ non-intersecting lattice paths with final displacement $2 \lambda$. Because for such self dual lattice paths $\tilde{\lambda}_{l}(j)=\tilde{\lambda}_{l}(2 n+1-j)$ (recall the discussion below (4.10) we have

$$
\prod_{j=1}^{2 n} q_{j}^{\frac{1}{2} \sum_{l=1}^{2 n} \tilde{\lambda}_{l}(j)}=\prod_{j=1}^{n}\left(q_{j} q_{2 n+1-j}\right)^{\frac{1}{2} \sum_{l=1}^{2 n} \tilde{\lambda}_{l}(j)} .
$$

This allows us to set

$$
q_{i}=q_{2 n+1-i} \quad(i=1, \ldots, n)
$$

and so with

$$
\tilde{s}_{2 \lambda}^{\text {s.d. }}\left(q_{1}, \ldots, q_{n}\right):=\left.\sum^{*} s_{2 \lambda}^{\text {s.d. }}\left(q_{1}, \ldots, q_{2 n}\right)\right|_{\substack{q_{i}=q_{2 n+1-i} \\(i=1, \ldots, n)}}=\prod_{j=1}^{n} q_{j}^{\sum_{l=1}^{2 n} \tilde{\lambda}_{l}(j)}
$$

(6.3) reads

$$
\prod_{i=1}^{n}\left(1-\alpha q_{i}\right) \prod_{i, j=1}^{n}\left(1-q_{i} q_{j}\right) \alpha^{\sum_{j=1}^{2 n}(-1)^{j-1} \lambda_{j}} \tilde{s}_{2 \lambda}^{\text {s.d. }}\left(q_{1}, \ldots, q_{n}\right) .
$$

As noted in 2], the polynomial $\tilde{s}_{2 \lambda}^{\text {s.d. }}$ is expressible in terms of Schur polynomials. To understand this point, one must first establish a relation between self dual lattice paths, represented as self dual tableaux, and domino tableaux. Regarding the latter, consider the diagram of a partition $2 \lambda$. Define a domino tableau, of shape $2 \lambda$ with content from $\{n+1, \ldots, 2 n\}$, as a tiling of the diagram by dominos with the dominos numbered from the set $\{n+1, \ldots, 2 n\}$ (each number repeated twice to fill the two squares of the domino) such that the number given to different dominos strictly increase down columns and 


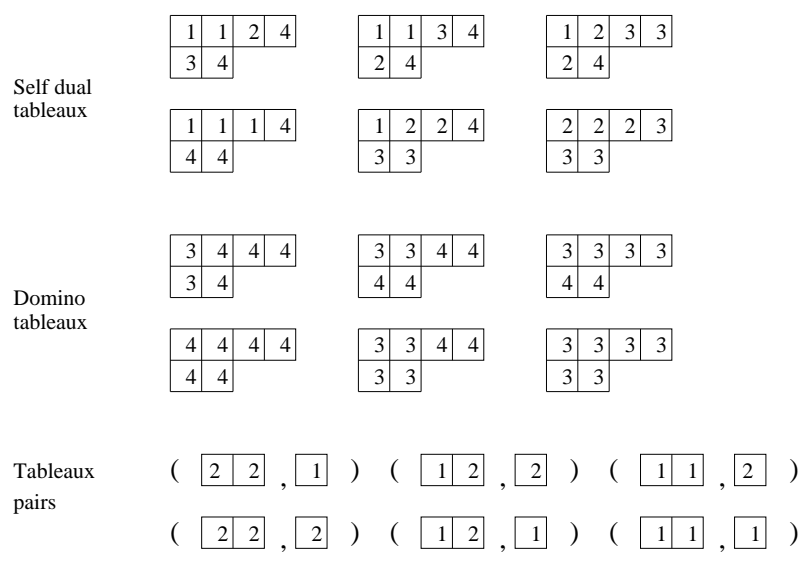

Figure 5: The self dual tableaux of shape 42 and content 4, the corresponding domino tableaux and the corresponding pair of tableaux of shape 2 , content 2 , and shape 1 , content 1.

weakly increase along rows. It is a known result (see e.g. 24]) that there is a bijection between self dual tableaux of shape $2 \lambda$, content $2 n$, and domino tableaux of shape $2 \lambda$ with content from $\{n+1, \ldots, 2 n\}$. In particular, to construct a domino tableau from a self dual tableau $P$ say, one applies in succession the Schützenberger evacuation operation (see e.g. [20]), and the operation of removing the last square displaced in this operation. The domino formed by the evacuated and removed squares is numbered by the number of the removed square, which will be between $n+1$ and $2 n$ (note that $P$ being self dual implies the sum of the entries of the evacuated and removed squares is $2 n+1$ ). The procedure is repeated until all dominos have been identified and numbered (see Figure 5 for an example of the end product of this mapping).

Having established the bijection between self dual tableaux and domino tableaux, one now makes use of a bijection between domino tableaux of shape $2 \lambda$ and content from $\{n+1, \ldots, 2 n\}$, and pairs of semi-standard tableaux $(P, Q)$ of shape $(\mu, \kappa)$ each of content $n$ with

$$
\text { shape } P=\left(\lambda_{1}, \lambda_{3}, \ldots, \lambda_{m}\right)=: \lambda^{+}, \quad \text { shape } Q=\left(\lambda_{2}, \lambda_{4}, \ldots, \lambda_{m-1}\right)=: \lambda^{-}
$$

where $m$ equals the length of $2 \lambda$ if the latter is odd, and one minus the length if it is even (and then $\lambda_{m}=0$ ). To construct $P(Q)$, remove all columns from the domino tableau for which the absolute value of the column number minus the length of the column is odd (even), and remove all even (odd) numbered rows. Finally subtract $n$ from each of the numbers (an example of the result of this mapping is given in Figure 5). Recalling the definition (6.5) of $\tilde{s}_{2 \lambda}^{\text {s.d. }}$ and the definition (2.4) of the Schur polynomials, it follows that

$$
\tilde{s}_{2 \lambda}^{\text {s.d. }}\left(q_{1}, \ldots, q_{n}\right)=s_{\lambda^{+}}\left(q_{1}, \ldots, q_{n}\right) s_{\lambda^{-}}\left(q_{1}, \ldots, q_{n}\right) .
$$

Consequently the probability (6.6) can be written as

$$
\prod_{i=1}^{n}\left(1-\alpha q_{i}\right) \prod_{i, j=1}^{n}\left(1-q_{i} q_{j}\right) \alpha^{\sum_{j=1}^{2 n}(-1)^{j-1} \lambda_{j}} s_{\lambda^{+}}\left(q_{1}, \ldots, q_{n}\right) s_{\lambda^{-}}\left(q_{1}, \ldots, q_{n}\right) .
$$

In the recent work [9] (6.9) was stated without derivation as being equal to the probability that the $2 n \times 2 n$ matrix $X$, symmetric about both the diagonal and anti-diagonal, and with elements distributed according to (6.1) and (6.2), maps under the RSK correspondence to a set of at most $2 n$ non-intersecting $\mathrm{u} / \mathrm{rh}$ lattice paths of final displacement $2 \lambda$. This is precisely the result derived here. 
From the definition (2.4) of the Schur polynomials in terms of non-intersecting lattice paths, it is easy to see that the well known identity

$$
\sum_{\lambda^{-}: \lambda^{+} \text {fixed }} \alpha^{\sum_{j=1}^{2 n}(-1)^{j-1} \lambda_{j}} s_{\lambda^{-}}\left(q_{1}, \ldots, q_{n}\right)=s_{\lambda^{+}}\left(q_{1}, \ldots, q_{n}, \alpha\right)
$$

holds. Thus the marginal probability of $\lambda^{+}$in (6.9) is equal to

$$
\prod_{i=1}^{n}\left(1-\alpha q_{i}\right) \prod_{i, j=1}^{n}\left(1-q_{i} q_{j}\right) s_{\lambda^{+}}\left(q_{1}, \ldots, q_{n}\right) s_{\lambda^{+}}\left(q_{1}, \ldots, q_{n}, \alpha\right) .
$$

This in turn implies that with

$$
L_{2 n}^{\otimes}:=\max \sum_{\substack{(1,1) \mathrm{u} / \mathrm{rh}(2 n, 2 n) \\ X=X^{T}=X^{R}}} x_{i, j}
$$

we have

$$
\begin{aligned}
& \operatorname{Pr}\left(L_{2 n}^{\otimes} \leq 2 l\right)=\operatorname{Pr}\left(L_{2 n}^{\otimes} \leq 2 l+1\right)=\prod_{i=1}^{n}\left(1-\alpha q_{i}\right) \prod_{i, j=1}^{n}\left(1-q_{i} q_{j}\right) \\
& \quad \times \sum_{\lambda^{+}: \lambda_{1} \leq l} s_{\lambda^{+}}\left(q_{1}, \ldots, q_{n}\right) s_{\lambda^{+}}\left(q_{1}, \ldots, q_{n}, \alpha\right) .
\end{aligned}
$$

The sum in (6.11) is a special case of that in 2.11) - thus replace $n \mapsto n+1$ in the latter and set $a_{i}=b_{i}=q_{i}(i=1, \ldots, n), a_{n+1}=0, b_{n+1}=\alpha$. It therefore follows from (2.3) that

$$
\begin{aligned}
& \operatorname{Pr}\left(L_{2 n}^{\bigotimes} \leq 2 l\right)=\operatorname{Pr}\left(L_{2 n}^{\otimes} \leq 2 l+1\right) \\
& \quad=\prod_{i=1}^{n}\left(1-\alpha q_{i}\right) \prod_{i, j=1}^{n}\left(1-q_{i} q_{j}\right)\left\langle\prod_{k=1}^{l}\left(1+\alpha e^{i \theta_{k}}\right) \prod_{j=1}^{n} \prod_{k=1}^{l}\left|1+q_{j} e^{i \theta_{k}}\right|^{2}\right\rangle_{U(l)}
\end{aligned}
$$

This result is the special case $\beta=0$ (the effect of setting $\beta=0$ is to constrain the elements on the anti-diagonal of $X$ to be even) of a result first derived in [2] using methods of symmetric function theory to sum over a $\beta$-generalization of (6.3).

\section{Matrices with a point reflection symmetry}

The point $(n+1 / 2, n+1 / 2)$ is at the centre of the region $1 \leq x, y \leq 2 n$. A point $(x, y)$ in this region reflected about this central point maps to the point $(2 n+1-x, 2 n+1-y)$. We thus say that a $2 n \times 2 n$ matrix $X$ has a point reflection symmetry (about the point $(n+1 / 2, n+1 / 2)$ ) if $x_{i, j}=x_{2 n+1-i, 2 n+1-j}$ $(i, j=1, \ldots, n)$ or consequently if $X=\left(X^{R}\right)^{T}$. For a matrix with this symmetry we can take as the independent elements the triangular region below the anti-diagonal $i<2 n+1-j(i, j=1, \ldots, 2 n)$ together with the portion of the anti-diagonal $i=2 n+1-j(i, j=1, \ldots, n)$.

We seek the constraint on the pairs of paths $\left(P_{1}, P_{2}\right)$, with both $P_{1}$ and $P_{2}$ of the same final displacements, which according to the RSK mapping are in correspondence with matrices $X$ with a point reflection symmetry. We have already noted that with $X$ mapping under $\operatorname{RSK}$ to $\left(P_{1}, P_{2}\right)$, $X^{R}:=\left[x_{2 n+1-j, 2 n+1-i}\right]_{i, j=1, \ldots, 2 n}$ maps to $\left(P_{2}^{R}, P_{1}^{R}\right)$ while $X^{T}:=\left[x_{j, i}\right]_{i, j=1, \ldots, 2 n}$ maps to $\left(P_{1}^{R}, P_{2}^{R}\right)$ and hence matrices with the point reflection symmetry $X=\left(X^{R}\right)^{T}$ map to a pair of $\mathrm{u} / \mathrm{rh}$ lattice paths of the same final displacements constrained so that

$$
P_{1}=P_{1}^{R}, \quad P_{2}=P_{2}^{R}
$$


We choose the independent entries of $X$ according to the geometric distribution

$$
\operatorname{Pr}\left(x_{i, j}=k\right)=\left(1-q_{i} q_{j}\right)\left(q_{i} q_{j}\right)^{k}
$$

where to be compatible with the point reflection symmetry we require $q_{2 n+1-i}=q_{i}(i=1, \ldots, n)$. With this specification it follows from the bijection that the probability $X$ maps to a pair of $\mathrm{u} / \mathrm{rh}$ lattice paths of final displacement $\mu$ is equal to

$$
\left(\prod_{i, j=1}^{n}\left(1-q_{i} q_{j}\right) \tilde{s}_{\mu}^{\text {s.d. }}\left(q_{1}, \ldots, q_{n}\right)\right)^{2} .
$$

We know from (6.8) that when the length of the parts of $\mu$ are all even, $\tilde{s}_{\mu}^{\text {s.d. }}$ can be given in terms of Schur polynomials. This is also true in the general case [24. One again proceeds by noting that there is a bijection between a general self dual tableaux of shape $\lambda$ and domino tableaux. An immediate consequence is that unless $\lambda$ admits a domino tiling — for which the necessary and sufficient condition is that the number of points $(i, j)$ in the diagram of $\lambda$ with $i+j$ even is equal to the number of points with $i+j$ odd - one has $\tilde{s}_{\mu}^{\text {s.d. }}=0$. It is also true that domino tableaux are in bijective correspondence with pairs of semi-standard tableaux of shape $\left(\mu^{(0)}, \mu^{(1)}\right),\left|\mu^{(0)}\right|+\left|\mu^{(1)}\right|=|\mu|$, each of content $n$ where $\mu^{(0)}$ and $\mu^{(1)}$ are the so called 2-quotient of the partition $\mu$. Regarding the latter, let $\mu$ be a partition of length $m$. Add to $\mu$ the partition $\delta_{m}:=(m-1, m-2, \ldots, 1,0)$, and from this construct two new partitions $\tilde{\mu}^{(0)}, \tilde{\mu}^{(1)}$ of lengths $m^{(0)}, m^{(1)}$, the first consisting of the even parts of $\mu+\delta_{m}$, and the second the odd parts of $\mu+\delta_{m}$. The 2-quotient is the pair of partitions $\mu^{(0)}, \mu^{(1)}$ specified by [17]

$$
\mu^{(0)}=\tilde{\mu}^{(0)} / 2-\delta_{m(0)}, \quad \mu^{(1)}=\left(\tilde{\mu}^{(1)}+1\right) / 2-\delta_{m^{(1)}} .
$$

Analogous to (6.8) one therefore has that if $\mu$ admits a domino tiling, then

$$
\tilde{s}_{\mu}^{\text {s.d. }}\left(q_{1}, \ldots, q_{n}\right)=\tilde{s}_{\mu^{(0)}}\left(q_{1}, \ldots, q_{n}\right) \tilde{s}_{\mu^{(1)}}\left(q_{1}, \ldots, q_{n}\right) .
$$

Regarding the converse of this statement, it's easy to see that the 2-quotient of a partition which admits a domino tiling is unique, while the 2-quotient of a partition which does not admit a domino tiling coincides with the 2-quotient of a partition which does. Hence, given arbitrary partitions $\mu^{(0)}, \mu^{(1)}$ there is a unique partition $\mu$ which admits a domino tiling and is such that (7.2) is satisfied. Furthermore, from the definition of a 2-quotient, if $\mu_{1} \leq 2 l$ then $\mu_{1}^{(0)} \leq l$ and $\mu_{1}^{(1)} \leq l$, while if $\mu_{1} \leq 2 l+1$ then $\mu_{1}^{(0)} \leq l+1$ and $\mu_{1}^{(1)} \leq l$, or $\mu_{1}^{(1)} \leq l+1$ and $\mu_{1}^{(0)} \leq l$. Thus with

$$
L_{2 n}^{\boxminus}:=\max \sum_{(1,1) \mathrm{u} / \operatorname{rh}(2 n, 2 n)} x_{i, j}
$$

we have

$$
\begin{aligned}
& \operatorname{Pr}\left(L_{2 n}^{\triangleright} \leq 2 l\right)=\prod_{i, j=1}^{n}\left(1-q_{i} q_{j}\right)^{2} \sum_{\mu: \mu_{1} \leq 2 l}\left(\tilde{s}_{\mu}^{\text {s.d. }}\left(q_{1}, \ldots, q_{n}\right)\right)^{2} \\
& \quad=\left(\prod_{i, j=1}^{n}\left(1-q_{i} q_{j}\right) \sum_{\kappa: \kappa_{1} \leq l}\left(s_{\kappa}\left(q_{1}, \ldots, q_{n}\right)\right)^{2}\right)^{2}=\left.\left(\operatorname{Pr}\left(L_{n}^{\square} \leq l\right)\right)^{2}\right|_{\left\{a_{i}\right\}=\left\{b_{i}\right\}=\left\{q_{i}\right\}}
\end{aligned}
$$

where the final equality follows upon comparison with (2.11), and

$$
\begin{aligned}
\operatorname{Pr}( & \left.L_{2 n}^{\triangleright} \leq 2 l+1\right)=\prod_{i, j=1}^{n}\left(1-q_{i} q_{j}\right)^{2} \sum_{\mu: \mu_{1} \leq 2 l+1}\left(\tilde{s}_{\mu}^{\text {s.d. }}\left(q_{1}, \ldots, q_{n}\right)\right)^{2} \\
& =\prod_{i, j=1}^{n}\left(1-q_{i} q_{j}\right)^{2} \sum_{\kappa: \kappa_{1} \leq l+1}\left(s_{\kappa}\left(q_{1}, \ldots, q_{n}\right)\right)^{2} \sum_{\kappa: \kappa_{1} \leq l}\left(s_{\kappa}\left(q_{1}, \ldots, q_{n}\right)\right)^{2} \\
& =\left.\left.\operatorname{Pr}\left(L_{n}^{\square} \leq l+1\right)\right|_{\left\{a_{i}\right\}=\left\{b_{i}\right\}=\left\{q_{i}\right\}} \operatorname{Pr}\left(L_{n}^{\square} \leq l\right)\right|_{\left\{a_{i}\right\}=\left\{b_{i}\right\}=\left\{q_{i}\right\}} .
\end{aligned}
$$


The results (7.3) and (7.4) were stated without derivation in [3]. The derivation given here uses the strategy outlined in [2] to derive (7.3) and (7.4) in the exponential limit which corresponds to the appropriately symmetrized form of the Hammersley process.

\section{References}

[1] J. Baik, P. Deift, and K. Johansson. On the distribution of the length of the longest increasing subsequence of random permutations. J. Amer. Math. Soc., 12:1119-1178, 1999.

[2] J. Baik and E.M. Rains. Algebraic aspects of increasing subsequences. Duke Math. J., 109:1-65, 2001.

[3] J. Baik and E.M. Rains. Symmetrized random permutations. In P.M. Bleher and A.R. Its, editors, Random matrix models and their applications, volume 40 of Mathematical Sciences Research Institute Publications, pages 171-208. Cambridge University Press, United Kingdom, 2001.

[4] A. Borodin, P.L. Ferrari, and T. Sasamoto. Transition between Airy 1 and Airy 2 processes and the TASEP fluctuations. arXiv:math-ph/0703023.

[5] P.J. Forrester. Log-gases and Random Matrices. Book in preparation.

[6] P.J. Forrester. Log-gases and Random Matrices. www.ms.unimelb.edu.au/ ${ }^{\sim}$ matpjf/matpjf.html.

[7] P.J. Forrester. Growth models, random matrices and Painlevé transcendents. Nonlinearity, 16:R27R49, 2003.

[8] P.J. Forrester and E.M. Rains. Correlations for superpositions and decimations of Laguerre and Jacobi orthogonal matrix ensembles with a parameter. Probab. Theory Relat. Fields, 130:518-576, 2004.

[9] P.J. Forrester and E.M. Rains. Interpretations of some parameter dependent generalizations of classical matrix ensembles. Probab. Theory Relat. Fields, 131:1-61, 2005.

[10] W. Fulton. Young Tableaux. London Mathematical Society Student Texts. CUP, Cambridge, 1997.

[11] I.M. Gessel. Symmetric functions and p-recursiveness. J. Comb. Th. A, 53:257-285, 1990.

[12] C. Greene. An extension of Schensted's theorem. Adv. in Math., 14:254-265, 1974.

[13] K. Johansson. Shape fluctuations and random matrices. Commun. Math. Phys., 209:437-476, 2000.

[14] K. Johansson. Non-intersecting paths, random tilings and random matrices. Prob. Theory Rel. Fields, 123:225-280, 2002.

[15] D.E. Knuth. Permutations, matrices and generalized Young tableaux. Pacific J. Math., 34:709-727, 1970.

[16] C. Krattenthaler. The major counting of nonintersecting lattice paths and generating functions for tableaux. volume 552 of Memoirs of the American Mathematical Society. American Mathematical Society, Providence, Rhode Island, 1995.

[17] I.G. Macdonald. Hall polynomials and symmetric functions. Oxford University Press, Oxford, 2nd edition, 1995.

[18] M.L. Mehta. Random Matrices. Academic Press, New York, 2nd edition, 1991. 
[19] E.M. Rains. Increasing subsequences and the classical groups. Elect. J. of Combinatorics, 5:\#R12, 1998.

[20] B.E. Sagan. The symmetric group. Springer-Varlag, New York, 2nd edition, 2000.

[21] H. Spohn. Exact solutions for KPZ-type growth processes, random matrices, and equilibrium shapes for crystals. arXiv:condmat/0512011, 2005.

[22] J.R. Stembridge. Non-intersecting paths, Pfaffians and plane partitions. Adv. Math., 83:96-131, 1990.

[23] G. Szegö. Orthogonal polynomials. American Mathematical Society, Providence R.I., 4th edition, 1975.

[24] van Leeuwen. The Robinson-Schensted and Schützenberger algorithms, an elementary approach. Elect. J. Combin., 3:R15, 1996. 